\title{
Intuitive Expertise and Irrelevant Options
}

\author{
Alex Wiegmann ${ }^{1,2^{*}}$, Joachim Horvath ${ }^{2 *}, \&$ Karina Meyer ${ }^{3}$ \\ ${ }^{1}$ Department of Cognitive Science, University of Göttingen, Germany \\ ${ }^{2}$ Institute for Philosophy II, Ruhr-University Bochum, Germany \\ ${ }^{3}$ Department of Educational Research, University of Göttingen, Germany
}

\begin{abstract}
In the so-called push dilemma, an out-of-control speed-train is about to run over five people and can only be stopped by pushing a heavy person onto the tracks. Most lay people and moral philosophers consider it morally wrong to kill the heavy person. Unger $(1992,1996)$, however, argued that adding irrelevant options to the push dilemma would overturn this intuition. In this paper, we empirically test Unger's claim with both lay people and expert moral philosophers. Including philosophical experts allowed us to investigate the so-called expertise defense, according to which the intuitions of philosophical experts are superior to the intuitions of lay people. Overall, we found that adding irrelevant options indeed increased the ratings for the "push option". Moreover, we found that the intuitions of expert moral philosophers were no less susceptible to the presence of irrelevant options than lay people's intuitions. We discuss how these findings bear on the expertise defense.

Keywords: moral judgment; moral intuitions; Unger; trolley dilemmas; experimental philosophy; expertise defense; irrelevant options; order effects
\end{abstract}

\section{Author Note:}

Correspondence concerning this article should be addressed to Dr. Alex Wiegmann, RuhrUniversität Bochum, Institut für Philosophie II, Universitätsstraße 150, 44801 Bochum, Germany. Email: awiegma@gwdg.de

* Alex Wiegmann and Joachim Horvath contributed equally to this paper and should be regarded as joint first authors. 


\section{Introduction}

Thought experiments play an important role in moral philosophy. Their main methodological purposes are to develop and evaluate moral theories. With regards to the developing aspect, moral dilemmas are used to trigger moral intuitions, and these intuitions can guide the moral philosopher towards a normative moral theory. ${ }^{1}$ As an example, consider the methodology of a leading moral philosopher, Frances Kamm (2007, p. 5):

Consider as many case-based judgments of yours as prove necessary. Do not ignore some case-based judgments, assuming they are errors, just because they conflict with simple or intuitively plausible principles that account for some subset of your case-based judgments. Work on the assumption that a different principle can account for all of the judgments. Be prepared to be surprised at what this principle is. Remember that this principle can be simple, even though it is discovered by considering many complex cases. (If the principle is complex, this would not undermine the claim that people have intuitive judgments in accord with it, since people need not be conscious of the principle to have case-based intuitive judgments.)

In a related way, thought experiments can be used to evaluate normative moral theories. If, for example, a normative moral theory is applied to concrete cases and tells us that the right action-according to the theory-stands in sharp contrast to our moral intuitions, this fact would strongly count against the theory. This kind of criticism constitutes one of the strongest arguments against consequentialist, especially utilitarian, moral theories (cf. Hooker, 2000).

Consequentialism tells us, roughly, that the morally right act is the one that has the best consequences-impartially considered. Although this principle may sound plausible in the abstract, it can sometimes prescribe actions that most lay people and moral philosophers consider morally wrong. One of the most popular thought experiments in this vein is a moral dilemma called push dilemma, or footbridge dilemma (cf. Thomson, 1985). In this kind of scenario, an out-of-control trolley is about to run over and thereby kill five people. The only possibility to save the five people consists in pushing a heavy stranger from a bridge onto the tracks, so that the heavy stranger, who will die in the collision, stops the trolley. However, most lay people (cf. Waldmann, Nagel, \& Wiegmann, 2012) and philosophers (cf. Schwitzgebel \& Cushman, 2012, 2015) consider this action as morally

\footnotetext{
${ }^{1}$ In this paper, we intend to use the term 'intuition' in a largely theory-neutral way. For example, we intend to stay neutral on whether intuitions in this sense are just ordinary judgments (see, e.g., Machery, 2017) or a distinctive kind of mental states (see, e.g., Bengson, 2015; Chudnoff, 2013). Keeping the label 'intuition' still seems helpful, because much of the relevant philosophical and metaphilosophical discussion is cast in this way.
} 
wrong-although it brings about the best consequences. Since this thought experiment is considered as a strong counterexample to consequentialist moral theories, it does not come as a surprise that supporters of consequentialism tried to debunk the widely shared intuition that pushing the heavy man would be morally wrong. For example, Unger $(1992,1996)$ claims that intuitions about the push dilemma are not robust and can be changed by adding more options. To support this claim, he first introduces the following case, labeled Heavy Skater:

By sheer accident, an empty trolley, nobody aboard, is starting to roll down a certain track. Now, if you do nothing about the situation, your first option, then, in a couple of minutes, it will run over and kill six innocents who, through no fault of their own, are trapped down the line (just beyond an "elbow" in the track). [...] Regarding their plight, you have one other option: Further up the track, near where the trolley's starting to move, there's a path crossing the main track and, on it, there's a very heavy man on roller skates. If you turn a remote control dial, you'll start up the skates, you'll send him in front of the trolley, and he'll be a trolley-stopper. But, the man will be crushed to death by the trolley he then stops. (Unger, 1996, p. 87)

Unger believes that most people consider redirecting the heavy skater as morally wrong. However, he also thinks that this intuition would change if more options were added to the scenario, as in the following case, which we will label Four Options (Unger, 1996, p. 90; see Figure 1):

By sheer accident, an empty trolley, nobody aboard, is starting to roll down a certain track. Now, if you do nothing about the situation, your first option, then, in a couple of minutes, it will run over and kill six innocents who, through no fault of their own, are trapped down the line. [...] Regarding their plight, you have three other options: On your second option, if you push a remote control button, you'll change the position of a switch-track, switch A, and, before it gets to the six, the trolley will go onto another line, on the left-hand side of switch A's fork. On that line, three other innocents are trapped and, if you change switch $A$, the trolley will roll over them. [...] On your third option, you'll flip a remote control toggle and change the position of another switch, switch B. Then, a very light trolley that's rolling along another track, the Feed Track, will shift onto B's lower fork. As two pretty heavy people are trapped in this light trolley, after going down this lower fork the vehicle won't only collide with the onrushing empty trolley, but, owing to the combined weight of its unwilling passengers, the collision will derail the first trolley and both trolleys will go into an uninhabited area. Still, the two trapped passengers will die in the collision. On the other hand, if you don't change switch B, the lightweight trolley will go along B's upper fork and, then, it will bypass the empty trolley, and its two passengers won't die soon. 
[...] Finally, you have a fourth option: Further up the track, near where the trolley's starting to move, there's a path crossing the main track and, on it, there's a very heavy man on roller skates. If you turn a remote control dial, you'll start up the skates, you'll send him in front of the trolley, and he'll be a trolley-stopper. But, the man will be crushed to death by the trolley he then stops.

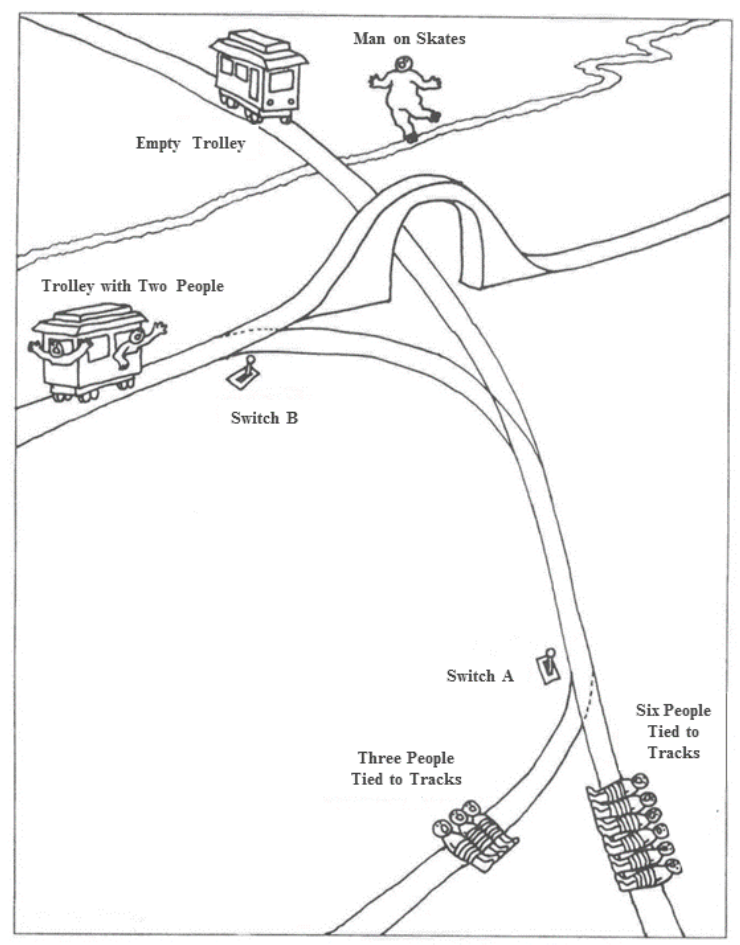

Figure 1. "Diagram for the Switches and the Skates" (original illustration of the several-option case). Reprinted by permission of Oxford University Press, USA from Living High and Letting Die: Our Illusion of Innocence (p. 89), by Peter Unger, 1996, New York: Oxford University Press.

In this several-option case, Unger claims most people would consider redirecting the heavy skater as the morally right thing to do.

Wiegmann and Meyer (2015) tested Unger's idea with a push dilemma (labeled Two Options; see Figure 2) and a several-option scenario (labeled Six Options; see Figure 2) that also included the option to push a heavy stranger from a bridge. ${ }^{2}$ These two scenarios were presented following each other in randomized order. The two main findings were: First, when one considers only the scenarios that were presented first, participants were more likely to choose the push option in Six Options than in Two Options. Second, participants' responses to the first scenario influenced their responses to the second scenario insofar as

\footnotetext{
${ }^{2}$ For another study testing Unger's claim, see Weijers (2015) and Weijers, Unger, and Sytsma (ms).
} 
they tended to choose the same option for both scenarios. For example, when participants received Two Options after Six Options, they were more likely to choose the push option in both scenarios than when Two Options was presented first.

Since adding intermediate options to the push dilemma arguably should not increase participants' moral preferences for pushing the heavy man from the bridge, the addition of intermediate options works as a morally irrelevant factor here (see e.g., Huber, Payne, \& Puto, 1982, for only one of several non-moral settings in which adding an option increases the choices for an option that has been available before; more on this in the Discussion section). Hence, the paradigm of two-option versus several-option cases provides a framework for testing whether the moral intuitions of lay people and experts are influenced by morally irrelevant factors. An Unger-style several-option scenario is also a less familiar version of the standard trolley cases, since it has triggered comparatively little discussion so far. $^{3}$ This may help to forestall worries about testing expert ethicists with all-too-familiar cases, which may not fully engage their intuitive expertise (cf. Horvath \& Wiegmann, 2016; Rini, 2015).

\section{Intuitive Expertise and the Expertise Defense}

Since intuitive expertise is a well-researched psychological phenomenon in its own right (see, e.g., Kahneman \& Klein, 2009), doing experiments on Unger's several-option cases with experts in moral philosophy would require no special motivation or justification. But as it happens, the issue of intuitive expertise also turns out to be vitally important for recent debates in philosophical methodology. These debates were mainly triggered by the new movement of experimental philosophy, which started its life in the early 2000s with an empirical investigation of intuitions about philosophical thought experiments, in particular

\footnotetext{
${ }^{3}$ Further evidence for this claim might be that Unger's original presentation of several-option cases in Causing and Preventing Serious Harm (1992) has only ten citations on Google Scholar, and Kamm's critical discussion Grouping and the Imposition of Loss (1998) has only two citations (as of September 6, 2018). It should be noted that the relevant material by Unger and Kamm was later included in monographs (Unger, 1996; Kamm, 2007) that have far higher citation rates. However, the issue of several-option cases does not seem to be among the more influential themes from these two books.
} 
by using survey-based methods from psychology and the social sciences (for an overview, see, e.g., Alexander, 2012; Sytsma \& Livengood, 2016). ${ }^{4}$

A number of critical findings from experimental philosophy suggest that lay people's intuitions about philosophical thought experiments vary with factors that are seemingly irrelevant to their proper evaluation, such as cultural background (Machery, Mallon, Nichols, \& Stich, 2004), affective content (Nichols \& Knobe, 2007), order of presentation (Liao, Wiegmann, Alexander, \& Vong, 2012; Swain, Alexander, \& Weinberg, 2008), or heritable personality traits (Feltz \& Cokely, 2009). On the basis of these and related findings, experimental philosophers of a "restrictionist" stripe have challenged the epistemic trustworthiness of intuitions about philosophical thought experiment cases (see, e.g., Alexander, Mallon, \& Weinberg, 2009; Alexander \& Weinberg, 2007; Feltz \& Cokely, 2012; Machery, 2017). If successful, this challenge might have far-reaching consequences for a number of key philosophical debates, for example, about knowledge or free will, since it is not clear how these debates should proceed without appeals to intuitions about thought experiments. $^{5}$

It is no wonder, then, that other philosophers have forcefully responded to the experimental restrictionist challenge in various ways (see, e.g., Horvath, 2010; Kauppinen, 2007; Ludwig, 2007; Nagel, 2012; Sosa, 2007, 2009). An especially interesting and muchdiscussed response is the so-called expertise defense (for recent surveys, see Horvath \& Wiegmann, 2016; Nado, 2014). The basic idea is that professional philosophers are experts concerning the intuitive evaluation of thought experiment cases. Based on this idea, problematic results about lay people's intuitions are regarded as irrelevant to philosophical practice, because professional philosophers are expected to be largely resistant to the influence of irrelevant factors on their thought experiment intuitions (see, e.g., Devitt, 2011; Hales, 2006; Horvath, 2010; Ludwig, 2007; Williamson, 2011).

There are different ways of motivating the expertise defense, and thus different ways of providing prima facie support for the crucial claim that the intuitions of professional

\footnotetext{
${ }^{4}$ However, experimental philosophy has broadened its scope considerably since its beginnings (see, e.g., Machery \& O'Neill, 2014; Sytsma \& Livengood, 2016).

${ }^{5}$ For skepticism about the methodological centrality of intuitions about thought experiments in philosophy, see Cappelen (2012) and Deutsch (2015); see Nado (2016) for a recent reply.
} 
philosophers are resistant to the influence of irrelevant factors, due to their intuitive expertise in evaluating thought experiment cases.

One way is by appealing to an analogy between professional philosophers and the professional practitioners of other disciplines, such as lawyers, mathematicians, or physicists (cf. Hales, 2006; Ludwig, 2007; Sorensen, 2014; Williamson, 2005, 2007, 2011). Since the latter are rightly regarded as experts in their field, philosophers should be regarded as experts in their own field as well, which typically includes the intuitive evaluation of thought experiment cases in their area of expertise.

Another way is to appeal to a general presumption of expertise for professional practitioners of any respectable intellectual discipline, including philosophy (cf. Horvath, 2010; Williamson, 2011). Although this general presumption is defeasible, even by empirical evidence, it would still suffice to shift the burden of proof to the critics of philosophers' intuitive expertise.

A third way of supporting the expertise defense appeals to specific cognitive skills of professional philosophers that might explain why their intuitions are less susceptible to the influence of irrelevant factors than those of lay people. For example, it seems plausible that professional philosophers are more sensitive to various conceptual distinctions that are crucial for the evaluation of thought experiment cases in their area of expertise (cf. Hofmann, 2010; Horvath, 2010; Ludwig, 2007; Williamson, 2007).

None of these ways of supporting the expertise defense is uncontroversial, and each faces problems and challenges of its own (for discussion, see Hitchcock, 2012; Horvath, 2010; Nado, 2015; Rini, 2014; Weinberg, Gonnerman, Buckner, \& Alexander, 2010). However, one point deserves special attention: What an argument in support of the expertise defense needs to show is not just that philosophers are experts in one sense or other-which they surely are-but rather that they have the quite specific intuitive expertise for evaluating philosophical thought experiments, an expertise of a kind that would make their intuitions largely resistant to the influence of irrelevant factors (Weinberg et al., 2010).

There are direct and indirect strategies for assessing this key claim about philosophers' intuitive expertise (Schulz, Cokely, \& Feltz, 2011). Indirect strategies, such as the one by Weinberg et al. (2010), rely on general findings about genuine expertise in other 
fields or disciplines (see, e.g., Ericsson, Charness, Feltovich, \& Hoffman, 2006). On that basis, they suggest various ampliative inferences from general findings about expertise to the alleged intuitive expertise of professional philosophers. A problem with indirect strategies of this kind is that paradigm cases of intuitive expertise in psychology, such as fire ground commanders (Klein, Calderwood, \& Clinton-Cirocco, 1986), or nurses in neonatal intensive care units (Crandall \& Getchell-Reiter, 1993), are strikingly disanalogous to thoughtexperimenting philosophers (cf. Horvath \& Wiegmann, 2016). So, at the present stage of the debate, direct strategies for assessing the expertise defense seem more promising, that is, strategies that rely on experimental studies with professional philosophers.

In light of the experimental restrictionist challenge, the most straightforward direct strategy would be to test whether the intuitions of professional philosophers are equally susceptible to the influence of irrelevant factors as those of lay people (for somewhat different approaches, see, e.g., Beebe \& Monaghan, 2018; Carter, Peterson, \& Bezooijen, 2015; Carter, Pritchard, \& Sheperd, ms; Horvath \& Wiegmann, 2016; Machery, 2012; Sytsma \& Machery, 2010). So far, there are only a few studies of this kind (Hitchcock \& Knobe, 2009; Schulz et al., 2011; Schwitzgebel \& Cushman, 2012, 2015; Tobia, Buckwalter, \& Stich, 2013; Tobia, Chapman, \& Stich, 2013), and we will briefly summarize their main results in the following.

To begin with, the study by Hitchcock and Knobe (2009) investigates intuitive judgments about actual causation, that is, judgments of the form "Peter caused the window to break". With respect to judgments of this kind, Hitchcock and Knobe found that "events that involve norm violations are especially likely to be selected as causes" (2009, p. 605), even by the 327 professional philosophers who took part in their study. Since facts about causation are often regarded as paradigms of objective facts - or at least as facts that are independent of normative considerations-, this result might indicate that expert intuitions about actual causation vary with the irrelevant factor of normative valence (however, the discussion by Hitchcock and Knobe suggests that this interpretation would be controversial).

The study by Schulz et al. (2011) indicates that the personality trait extraversionmore precisely, the facet warmth of this personality trait-predicts compatibilist intuitions about free will even in people who are familiar with the philosophical debate about free will. However, their "Free Will Skill Test" mainly tracks rather superficial or merely historical 
knowledge about the philosophical debate, such as whether "Arthur Schopenhauer said that there is definitely free will" (2011, p. 1730). At best, the Free Will Skill Test thus helps to exclude participants who have little or no familiarity with the philosophical debate about free will. It is doubtful whether the study really investigates what seems most relevant for the expertise defense, namely, the intuitive expertise of professional philosophers who specialize on free will.

Schwitzgebel and Cushman (2012) present evidence that order effects about ethical cases are about as large in professional philosophers and expert ethicists as in lay people (inter alia, they studied trolley cases that are similar to those we used in our own experiment-see below). In addition, they found that professional philosophers-even those who specialize on ethics - were more likely than lay people to endorse general moral principles that are in line with their order-sensitive judgments about particular cases. Schwitzgebel and Cushman suggest that this finding might be explained by philosophers' stronger tendency for post-hoc rationalization.

In a follow-up study, Schwitzgebel and Cushman (2015) replicated their earlier finding that the intuitive case judgments of expert ethicists are no less sensitive to order effects than those of lay people. Their follow-up study also addresses a number of worries about their earlier study, for example, that the antecedent familiarity of expert ethicists with trolley cases or the lack of reflection in standard experimental settings might have a significant effect on their results. However, Schwitzgebel and Cushman found that neither a forced delay in combination with the appeal to reflect on the scenario, nor antecedent familiarity with the relevant philosophical issues, nor self-reported stability of relevant views, and not even self-reported expertise on the specific issues in question had any tendency to reduce order effects in expert ethicists.

Tobia, Buckwalter, et al. (2013) found that professional philosophers respond differently to various ethical cases depending on whether the scenario is described in a firstperson or third-person way-an instance of the so-called actor-observer bias. For example, they investigated a standard trolley case where one option is to do nothing - and five people will be killed-and the other option is to direct the trolley to another track-and only one person will be killed. Professional philosophers' agreement that redirecting the trolley is permissible was much higher in the first-person condition (89\%) than in the third-person 
condition (64\%). Strikingly, this was almost the exact reversal of the ratings of lay people, who exhibited a higher rate of agreement in the third-person condition (90\%) than in the first-person condition (65\%).

The study by Tobia, Chapman, et al. (2013) confirms the susceptibility of professional philosophers' moral intuitions to actor-observer bias, although with a set of different, "purity-related" scenarios. In addition to that, they also found that even the ratings of professional philosophers were significantly influenced by the smell of Lysol, which is strongly associated with cleanliness. The direction of the effect was once again somewhat different in professional philosophers than in lay people.

To conclude, the summarized studies do not lend much support to the expertise defense, and all of them raise at least a modicum of doubt about the intuitive expertise of professional philosophers. This may look like a devastating result for the expertise defense. However, so far only a handful of studies bear directly on the question whether professional philosophers' intuitions are more resistant to the influence of irrelevant factors than those of lay people. Therefore, more work is needed before we can draw any robust conclusions about the precise contours and limitations of philosophers' intuitive expertise (see also Buckwalter, 2016; De Cruz, 2015; Horvath \& Wiegmann, 2016; Nado, 2015).

Our present study provides some of this additional work, and it does not turn out to be good news for the expertise defense. Basically, our study confirms the growing experimental consensus that the moral intuitions of philosophical experts are no less susceptible to order effects than those of lay people. Moreover, we have identified a new irrelevant factor that influences the intuitions of expert ethicists to a comparable or even greater degree than those of lay people: the availability of irrelevant additional options.

\section{Experiment}

The data is available online here: https://osf.io/8ycfh/

\subsection{Participants}

Overall, the data of 279 participants were included in our main analysis. The mean age was 34 years, $62 \%$ identified as male, $34 \%$ as female, and the remaining participants as other or prefer not to say. In this sample, 134 participants were identified as expert participants 
(mean age was 36 years; $68 \%$ identified as male, $25 \%$ as female, and $7 \%$ as other or prefer not to say) and 145 as lay participants (mean age 31 years; $57 \%$ male, $43 \%$ female).

Our expert participants were recruited via a call for participation that included a link which directed the participants to the online experiment. The call was distributed on an electronic mailing list for philosophers (PHILOS-L), on the Experimental Philosophy Blog (http://philosophycommons.typepad.com/xphi/), and on PEA Soup (http://peasoup.us), a blog that specializes on ethics. In order to be included as expert participants, participants had to indicate, first, that they have a PhD (64\%) or MA (36\%) in philosophy and, second, that moral philosophy or ethics is one of their areas of specialization (62\%) or competence (38\%). Furthermore, we excluded the data of participants who did not complete the survey, completed it in less than 90 seconds, or failed to answer an easy transitivity correctly. Out of initially 278 participants, 134 met these criteria, which were not mentioned in the call for participation or during the survey.

Lay participants were recruited via a database located in the UK (Prolific Academics; cf. Palan \& Schitter, 2017). Each participant received a compensation of $£ 0.50$. To ensure that lay participants differ strongly from expert participants in their level of philosophical expertise, we only included the data of participants who indicated that they had no prior experience with philosophy, or only some experience in school but not in university, and who had not participated in a trolley-style experiment before. Moreover, we excluded the data of participants who did not complete the survey, completed it in less than 90 seconds, or failed to answer an easy transitivity correctly. Out of initially 485 participants, 145 met these criteria, which were not mentioned in the call for participation or during the survey. ${ }^{6}$

\subsection{Design and Procedure}

The experiment was conducted on the Internet. Upon clicking on a link that participants received via email or in a blog post (experts), or on the Prolific Academics page (lay people), they were redirected to a website with the experiment. Participants were first presented with general instructions. These instructions familiarized them with the question mode, asked them to read the subsequent case descriptions carefully, and appealed to them to take the task seriously. After that, participants were randomly assigned to one of two

\footnotetext{
${ }^{6}$ The vast majority of excluded lay participants were excluded because they indicated that they had participated in a trolley-style experiment before.
} 
conditions. In one condition, they saw Two Options first and then Six Options, and in the other condition, they saw Six Options first and then Two Options. Here is the exact wording of Two Options:

On the test ground of a modern railroad property an unmanned speed-train (that normally can be remote-controlled) is out of control due to a technical defect. This speedtrain is heading towards nine railroad workers that are maintaining the tracks. ${ }^{7}$ Since these workers are wearing a new type of hearing protection, they would not notice the speed-train on time and hence would be run over by it. Carl, an employee of the rail track control center, recognizes the upcoming accident. However, it is not possible to stop the train on time any more.

Carl can choose between exactly two options:

Option 1: Carl could run to a nearby bridge on which a heavy worker is standing and push this worker from the bridge. Thereby this worker would fall on the tracks and collide with the speed-train. Due to the collision with the heavy worker (Carl himself would not be heavy enough to stop the train) the speed-train would stop before it reaches the nine workers. The heavy worker would lose his life due to the collision.

Option 2: Carl could do nothing. In this case the nine workers would be run over by the speed-train and lose their lives in this accident.

And here is the exact wording of Six Options:

On the test ground of a modern railroad property an unmanned speed-train (that normally can be remote-controlled) is out of control due to a technical defect. This speedtrain is heading towards nine railroad workers that are maintaining the tracks. Since these workers are wearing a new type of hearing protection, they would not notice the speedtrain on time and hence would be run over by it. Carl, an employee of the rail track control center, recognizes the upcoming accident. However, it is not possible to stop the train on time any more.

Carl can choose between exactly six options:

Option 1: Carl could run to the nearby, left bridge on which a heavy worker is standing and push this worker from the bridge. Thereby this worker would fall on the tracks and collide with the speed-train. Due to the collision with the heavy worker (Carl himself would not be heavy enough to stop the train) the speed-train would stop before it reaches the nine workers. The heavy worker would lose his life due to the collision.

Option 2: Carl could push a button that would open a trap door and thereby causing two workers on top of the right bridge to fall on the tracks. The speed-train would collide with the two workers and be stopped before it reaches the nine workers. The two workers would lose their lives due to the collision.

Option 3: Carl could throw Switch 3 and thereby redirect a train carrying three workers from the lower parallel track onto the main track. The speed-train would collide with this

\footnotetext{
${ }^{7}$ Usually, only five lives are at stake in trolley-style cases. We raised the number to nine lives to keep it parallel to the Six Option case, in which we need a higher number to implement a variety of intermediate options, as suggested by Unger (see above). Moreover, the net number of lives that can be saved by redirecting the train (Option 5 below) is still four lives (5 versus 9 lives), which is the standard in the literature.
} 
train and be stopped before it reaches the nine workers. The three workers on the train would lose their lives due to the collision.

Option 4: Carl could throw Switch 2 and thereby redirect an empty train from the upper parallel track onto the main track. The speed-train would collide with this train and be stopped before it reaches the nine workers. On its way to the main track the empty train would run over four workers (wearing the novel hearing protection). The four workers would lose their lives due to the collision.

Option 5: Carl could throw Switch 1 and thereby redirect the speed-train from the main track onto a parallel track before it reaches the nine workers. On the parallel track the speed train would run over five workers (wearing the novel hearing protection). The five workers would lose their lives due to the collision.

Option 6: Carl could do nothing. In this case the nine workers would be run over by the speed-train and lose their lives in this accident.

Both case descriptions were accompanied by an illustration (see Figure 2 ) of the respective initial situation. The test question asked participants to indicate which of the available options Carl should choose. On the final page, we asked a number of demographic questions and questions concerning the participants' level of philosophical education and expertise.
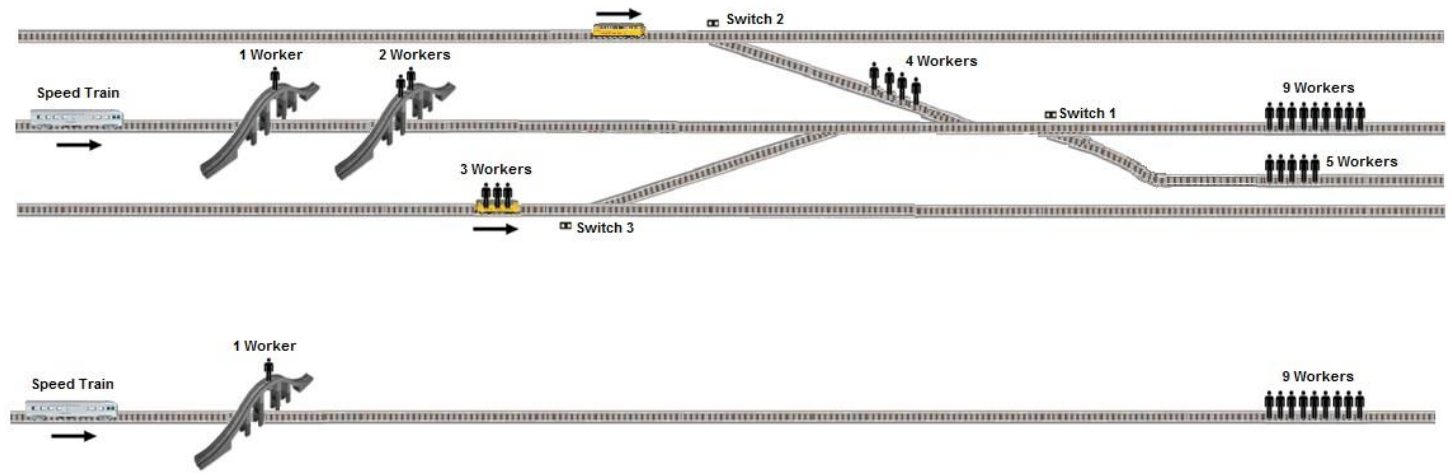

Figure 2. Illustration of the six-option case (on top) and the two-option case (below).

\subsection{Results}

The results are summarized in Figure 3 and Table 1 below. 


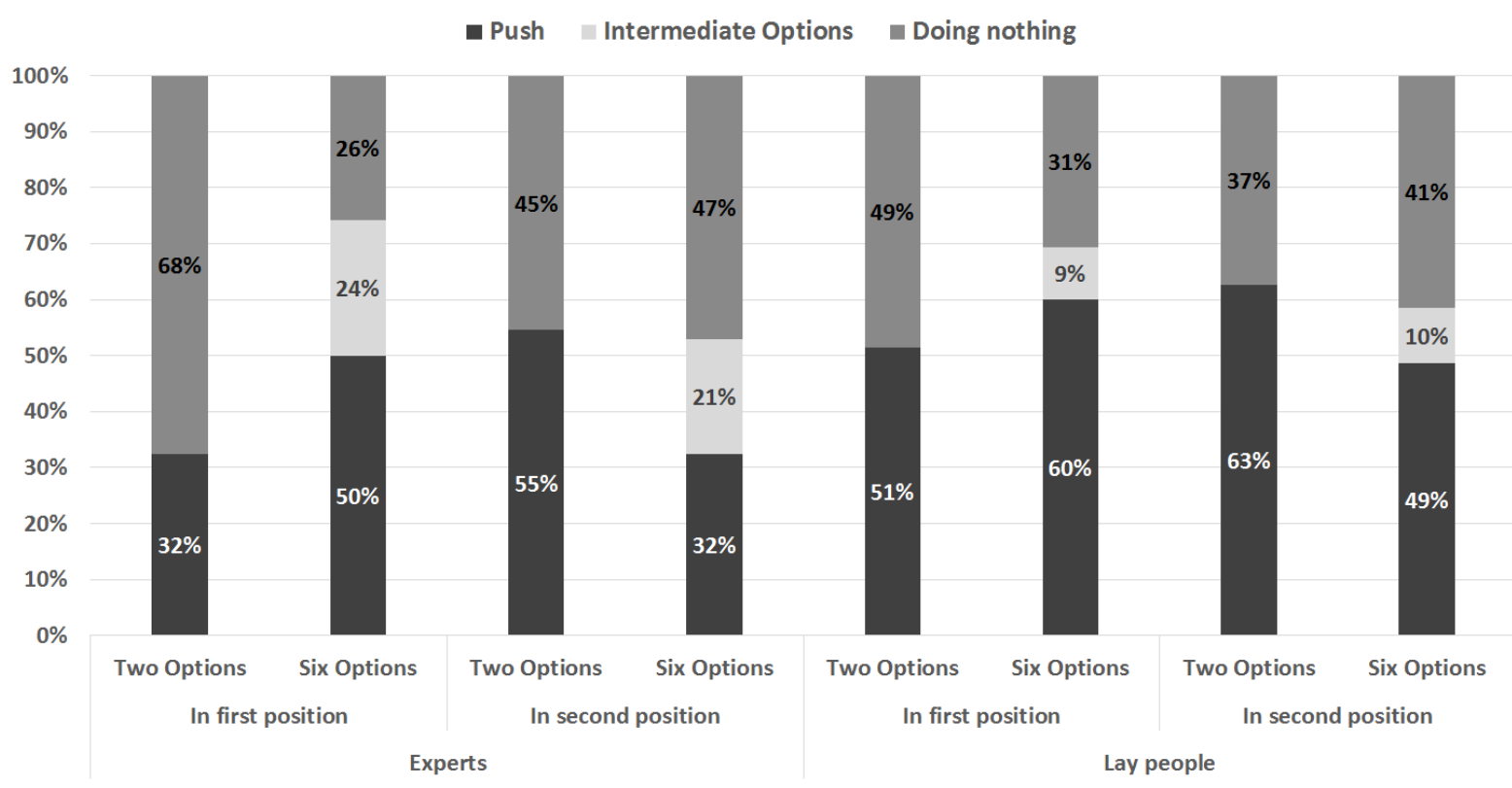

Figure 3. Participants' choices as a function of scenario, order of presentation, and level of expertise.

Table 1 Participants' Choices (Absolute Numbers) as a Function of Scenario, Order of Presentation, and Level of Expertise

\begin{tabular}{lcccccccc}
\hline & \multicolumn{4}{c}{ Experts } & \multicolumn{5}{c}{ Lay people } \\
\hline & \multicolumn{2}{l}{ In first position } & \multicolumn{2}{c}{ In second position } & \multicolumn{2}{l}{ In first position } & \multicolumn{2}{l}{ In second position } \\
\cline { 2 - 9 } & Two & Six & Two & Six & Two & Six & Two & Six \\
& Options & Options & Options & Options & Options & Options & Options & Options \\
\cline { 2 - 9 } Push & 22 & 33 & 36 & 22 & 36 & 45 & 47 & 34 \\
Doing nothing & 46 & 17 & 30 & 32 & 34 & 23 & 28 & 29 \\
Option 2 & - & 2 & - & 1 & - & 2 & - & 4 \\
Option 3 & - & 3 & - & 3 & - & 3 & - & 1 \\
Option 4 & - & 4 & - & 1 & - & 0 & - & 1 \\
Option 5 & - & 7 & - & 9 & - & 2 & - & 1 \\
\hline
\end{tabular}

\subsubsection{All Participants}

\subsubsection{Effect of Additional Options}

Two Options first vs. Six Options first. Before we compare the number of participants who chose the push option, as compared to doing nothing, in Two Options presented first versus Six Options presented first, we need to decide whether and how we take the intermediate options in Six Options into account. Depending on one's first-order moral views, the intermediate options could maybe be divided and simply added to the two extreme options for the purposes of analysis in one way or another. For instance, Frances Kamm (2007) 
would presumably only consider doing nothing and redirecting the threatening trolley as morally permissible. Hence, one could add the numbers for doing nothing and redirecting the trolley on the one side (and label this category as permissible options), and the numbers for all other options on the other side (and label this category as impermissible options). Then, one could analyze whether the proportions of participants choosing permissible versus impermissible options differ between Two Options and Six Options.

To avoid such tricky classification issues, we will perform the most conservative analysis, namely adding all choices of intermediate options to the option of doing nothing, and then testing whether the proportion of participants choosing the push option is still significantly higher in Six Options than in Two Options. This approach stacks the deck in favor of the participants and thus provides an unambiguous measure for testing whether their judgments exhibit an irrational pattern, namely, an increase of the ratings for the push option if more competing options are available (see section 4.1 for discussion).

When participants were presented with Two Options first, 42\% (58 out of 138) chose the push option, while $58 \%$ (80 out of 138) preferred not to push. When Six Options was presented first, $55 \%$ of the participants chose the push option (78 out of 141 ) and $45 \%$ (63 out of 141) preferred not to push. We built a binary logistic regression model to assess the influence of level of expertise, the number of available options, and the interaction thereof. The final model $\left(\chi_{2, N=279}^{2}=10.93, p=.004\right)$ is described in Table 2 and provides suggestive evidence that level of expertise and number of options have an influence on choosing or not choosing the push option, with philosophers being less likely to choose the push option, as compared to lay subjects, and participants being more likely to choose the push option in Six Options than in Two Options ( $p=.015$ for level of expertise and $p=.029$ for number of options). We arrived at this model by first including the level of expertise variable, which improved the model significantly, as compared to the baseline model, $\chi_{1, \mathrm{~N}=279}^{2}=6.14, p=$ .013. Adding the variable for number of options further improved the model, $\chi_{1, N=279}^{2}=4.78$, $p=.029$. Extending the model by including the interaction of these two variables, however, did not result in a significant model improvement, $\chi_{1, N=279}^{2}=0.63, p=.427$, suggesting that the influence of the number of options does not differ between lay and expert subjects. 
Coefficients of the Model Predicting whether a Participant Chooses the Push Option

\begin{tabular}{|c|c|c|c|c|c|c|c|}
\hline \multirow[b]{2}{*}{ Included } & B & S.E. & Wald & df & Sig. & $\operatorname{Exp}(B)$ & 95\% C.I.for $\operatorname{Exp}(B)$ \\
\hline & & & & & & & \\
\hline Level of expertise & -0.595 & 0.245 & 5.914 & 1 & 0.015 & 0.552 & {$[0.342 ; 0.891]$} \\
\hline Number of options & 0.532 & 0.244 & 4.741 & 1 & 0.029 & 1.702 & {$[1.055 ; 2.749]$} \\
\hline Constant & -0.036 & 0.209 & 0.029 & 1 & 0.865 & 0.965 & \\
\hline
\end{tabular}

\subsubsection{Order Effects}

Two Options. When participants were presented with Two Options first, $42 \%$ (58 out of 138) chose the push option, while 58\% (80 out of 138) preferred doing nothing. When Two Options was presented after Six Options, 59\% (83 out of 141) chose the push option, while $41 \%$ (58 out of 141) preferred doing nothing. We again built a binary logistic regression model to assess the influence of level of expertise, order of presentation, and the interaction thereof. The final model $\left(\chi_{2, N=279}^{2}=13.22, p=.001\right)$ is described in Table 3 and provides suggestive evidence that level of expertise and order of presentation both have an influence on choosing or not choosing the push option in Two Options, with philosophers being less likely to choose the push option in Two Options, as compared to lay subjects, and participants being more likely to choose the push option in Two Options if Two Options is presented after Six Options ( $p=.022$ for level of expertise and $p=.006$ for order of presentation). We arrived at this model by first including the variable for level of expertise, which improved the model significantly, as compared to the baseline model, $\chi_{1, \mathrm{~N}=279}^{2}=5.44$, $p=.02$. Adding the variable for order of presentation further improved the model, $\chi_{1, N=279}^{2}=$ $7.78, p=.005$. Extending the model by including the interaction of these two variables, however, did not result in a significant model improvement, $\chi_{1, N=279}^{2}=0.87, p=.351$, suggesting that the influence of order of presentation for choosing the push option in Two Options does not differ between lay and expert subjects.

Table 3

Coefficients of the Model Predicting Whether a Participant Chooses the Push Option in Two Options

\begin{tabular}{lccccccc}
\hline & B & S.E. & Wald & df & Sig. & $\operatorname{Exp(B)}$ & 95\% C.I.for Exp(B) \\
\cline { 2 - 8 } Included & & & & & & & \\
\cline { 1 - 7 } Level of expertise & -0.561 & 0.246 & 5.224 & 1 & 0.022 & 0.571 & {$[0.353 ; 0.923]$} \\
Position & 0.679 & 0.245 & 7.665 & 1 & 0.006 & 1.972 & {$[1.219 ; 3.19]$} \\
\hline
\end{tabular}




$-0.051$

0.209

0.06

$1 \quad 0.805$

0.95

Note. $R^{2}=0.03$ (Hosmer \& Lemeshow), 0.05 (Cox \& Snell), 0.06 (Nagelkerke). Model $\chi_{2, N=279}^{2}=13.22$, $p=.001$. Reference class for level of expertise: lay subjects, and for position: first position.

Six Options. When participants were presented with Six Options first, 55\% (78 out of 141) chose the push option, while $28 \%$ (40 out of 141) preferred doing nothing and $16 \%$ ( 23 out of 141) chose an intermediate option. When Six Options was presented after Two Options, 41\% (56 out of 138) chose the push option, while 44\% (61 out of 138) preferred doing nothing and $15 \%$ (21 out of 138) chose an intermediate option. To assess whether the order of presentation has an influence on participants moral judgments we will use the same classification for the intermediate options as we did before, namely, treating the push option as one category and all other options as the other category and then testing whether the proportion of participants choosing the push option differs as a function of order of presentation.

We again built a binary logistic regression model to assess the influence of level of expertise, order of presentation, and the interaction thereof. The final model $\left(\chi_{2, N=279}^{2}=\right.$ 10.99, $p=.004$ ) is described in Table 4 and provides suggestive evidence that level of expertise and order of presentation both have an influence on choosing or not choosing the push option in Two Options, with philosophers being less likely to choose the push option in Six Options, as compared to lay subjects, and participants being more likely to choose the push option in Six Options if Six Options is presented before Two Options ( $p=.028$ for level of expertise and $p=.015$ for order of presentation). We arrived at this model by first including the variable for level of expertise, which improved the model significantly, as compared to the baseline model, $\chi_{1, N=279}^{2}=5.06, p=.025$. Adding the variable for order of presentation further improved the model, $\chi_{1, N=279}^{2}=5.93, p=.015$. Extending the model by including the interaction of these two variables, however, did not result in a significant model improvement, $\chi_{1, N=279}^{2}=0.315, p=.575$, suggesting that the influence of order of presentation for choosing the push option in Two Options does not differ between lay and expert subjects.

Table 4

Coefficients of the Model Predicting Whether a Participant Chooses the Push Option in Six Options

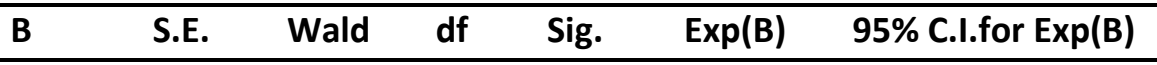




\begin{tabular}{|c|c|c|c|c|c|c|c|}
\hline \multirow{2}{*}{ Included } & \multirow[b]{3}{*}{-0.539} & \multirow{3}{*}{0.245} & \multirow[b]{3}{*}{4.846} & \multirow[b]{3}{*}{1} & \multirow[b]{3}{*}{0.028} & \multirow[b]{3}{*}{0.583} & \multirow[b]{3}{*}[0.361;0.943]{} \\
\hline & & & & & & & \\
\hline Level of expertise & & & & & & & \\
\hline Position & -0.592 & 0.245 & 5.865 & 1 & 0.015 & 0.553 & {$[0.342 ; 0.893]$} \\
\hline Constant & 0.470 & 0.208 & 5.115 & 1 & 0.024 & 1.599 & \\
\hline
\end{tabular}

Note. $R^{2}=0.03$ (Hosmer \& Lemeshow), 0.04 (Cox \& Snell), 0.05 (Nagelkerke). Model $\chi 2_{2, N=279}=10.99$, $p=.004$. Reference class for level of expertise: lay subjects, and for position: first position.

\subsubsection{Expert Participants}

\subsubsection{Effect of Additional Options}

Two Options first vs. Six Options first. We will use the same classification for the intermediate options as we did before, namely, treating the push option as one category and all other options as the other category. When expert participants were presented with Two Options first, $32 \%$ of them ( 22 out of 68 ) chose the push option, while $68 \%$ (46 out of 68 ) preferred not to push. When Six Options was presented first, $50 \%$ of the expert participants chose the push option (33 out of 66) and 50\% (33 out of 66) preferred not to push. This difference was significant, $\chi_{1, N=134}^{2}=4.31, p=.038, V=.18$, providing suggestive evidence that expert participants are more likely to choose the push option, as compared to doing nothing, in Six Options first.

\subsubsection{Order Effects}

Two Options. When expert participants were presented with Two Options first, 32\% (22 out of 68 ) chose the push option, while $68 \%$ (46 out of 68 ) preferred doing nothing. When Two Options was presented after Six Options, 55\% (36 out of 66) chose the push option, while $45 \%$ (30 out of 66 ) preferred doing nothing. This difference was statistically significant, $\chi_{1}^{2}$, $N=134=6.72, p=.01, V=.22$, providing suggestive evidence that presenting Six Options first increases the probability of participants choosing the push option in a subsequent presentation of Two Options.

Six Options. When expert participants were presented with Six Options first, 50\% (33 out of 66) chose the push option, while $26 \%$ (17 out of 66) preferred doing nothing and $24 \%$ (16 out of 66) chose an intermediate option. When Six Options was presented after Two Options, $32 \%$ (22 out of 68 ) chose the push option, while $47 \%$ (32 out of 68 ) preferred doing nothing and $21 \%$ (14 out of 68 ) chose an intermediate option. 
To assess whether participants' choices in Six Options are affected by being presented with Two Options first, we will use the same classification for the intermediate options as we did before, namely, treating the push option as one category and all other options as the other category and then testing whether the proportion of participants choosing the push option differs as a function of order of presentation. ${ }^{8}$ When Six Options was presented first, $50 \%$ of the expert participants chose the push option (33 out of 66 ), while also $50 \%$ (33 out of 66) preferred not to push. When Six Options was presented second, 32\% of the expert participants chose the push option (22 out of 68 ), while $68 \%$ (46 out of 68 ) preferred not to push. This difference was significant, $\chi^{2}{ }_{1, N=134}=4.31, p=.038, V=0.18$, providing suggestive evidence that presenting Two Options first decreases the probability of expert participants choosing the push option in a subsequent presentation of Six Options. ${ }^{9}$

\subsubsection{Lay Participants}

\subsubsection{Effect of Additional Options}

Two Options first vs. Six Options first. First, we will use the same classification for the intermediate options as we did before, namely, treating the push option as one category and all other options as the other category. When lay participants were presented with Two Options first, $51 \%$ of them (36 out of 70 ) chose the push option, while $49 \%$ (34 out of 70 ) preferred doing nothing. When Six Options was presented first, $60 \%$ of the lay participants chose the push option (45 out of 75 ) and $40 \%$ (30 out of 75 ) preferred doing nothing. This increase in choosing the push option in Six Options was not significant, $\chi_{1, N=145}^{2}=1.08, p=$ $.30, V=.09$.

\subsubsection{Order Effects}

Two Options. When lay participants were presented with Two Options first, 51\% (36 out of 70) chose the push option, while $49 \%$ (34 out of 70 ) chose doing nothing. When Two Options

\footnotetext{
${ }^{8}$ If we include the intermediate options as a third category, the same inference could be drawn $\chi_{2, N=134}^{2}=6.90$, $p=.031, V=.16)$, namely, that the data provide suggestive evidence that order of presentation affected participants' choices.

${ }^{9}$ The fact that the statistical values for the comparison of Six Options first versus Six Options second are identical with the ones for the comparison of Two Options first versus Six Options first does not seem to be a coincidence. A closer look at individual responses revealed that philosophers were perfectly consistent in their responses when first judging Two Options and then Six Options: Every single philosopher who chose the push option in Two Options also chose the push option in Six Options, and whenever a philosopher did not choose the push option in Two Options, she did not do so in Six Options either. This perfectly consistent pattern suggests that philosophers might have expertise in giving consistent responses (see also Discussion).
} 
was presented after Six Options, 63\% (47 out of 75 ) chose the push option ${ }^{10}$, while $37 \%$ (28 out of 75) preferred doing nothing. This difference was not significant, $\chi_{1, N=145}^{2}=1.87, p=$ $.17, V=.11$.

Six Options. When lay participants were presented with Six Options first, 60\% (45 out of 75) chose the push option, while 31\% (23 out of 75 ) preferred doing nothing and $9 \%$ (7 out of 75) chose an intermediate option. When Six Options was presented after Two Options, 49\% (34 out of 70 ) chose the push option, while $41 \%$ (29 out of 70 ) preferred doing nothing and $10 \%$ (7 out of 70 ) chose an intermediate option.

To assess whether participants' choices in Six Options are affected by being presented with Two Options first, we will use the same classification for the intermediate options as we did before, namely, treating the push option as one category and all other options as the other category, and then test whether the proportion of participants choosing the push option differs as a function of order of presentation. ${ }^{11}$ When Six Options was presented first, $60 \%$ of the lay participants chose the push option (45 out of 75 ), while $40 \%$ (30 out of 75 ) preferred not to push. When Six Options was presented after Two Options, 49\% (34 out of 70) chose the push option, while $51 \%$ (36 out of 70 ) preferred doing nothing. This difference was not significant, $\chi_{1, N=145}^{2}=1.91, p=.167, V=.11$.

\section{Discussion}

Let us begin by discussing the general findings for all participants before we focus on expert participants in particular.

As Unger $(1992,1996)$ predicted, we found that adding intermediate options to the push dilemma increased the number of participants who preferred the push option. Whereas doing nothing was preferred to the push option in Two Options (when presented first), the latter was preferred to the non-push options in Six Options (when presented first).

\footnotetext{
${ }^{10}$ The proportion of participants choosing the push option (51\%) is relatively high, especially in comparison with earlier seminal findings ( $10 \%$ in Mikhail, 2007). However, the numbers in more recent studies are usually higher (e.g. 40\% in Capraro \& Sippel, 2017), and Cao et al. (2017) recently found that participants preferred the push option in a two-option scenario when 15 lives were at stake. In light of these findings, we speculate that our relatively high numbers are based on the fact that 9 lives instead of 5 lives-as usual-were at stake. ${ }^{11}$ If we include the intermediate options as a third category, the same inference could be drawn $\chi_{2, N=145}^{2}=$ $2.05, p=.36, V=.08$ ), namely, that the data provide no evidence that order of presentation affected participants' choices.
} 
Given the main purpose of this paper and the fact that we have not conducted further experiments that would allow us to identify the underlying psychological mechanisms, we will refrain from speculating about the psychological explanation of this effect (see, e.g., Huber et al., 1982 for an explanation of a classic effect of this kind).

We also found an effect of order of presentation, which was already predicted by Unger $(1992,1996)$ as well. When Two Options was presented first, fewer participants chose the push option, as compared to when Two Options was presented after Six Options. And when Six Options was presented first, more participants chose the push option, as compared to when Six Options was presented after Two Options.

Concerning the best psychological explanation for order effects in moral judgments, several proposals have been put forward (cf. Horne, Powell, \& Spino, 2013; Lombrozo, 2009; Schwitzgebel \& Cushman, 2012; Wiegmann \& Waldmann, 2014). Since the design of our experiment does not allow us to identify the underlying psychological mechanisms or to decide between competing explanations, we will largely avoid this discussion here.

However, the specific setup of our experiment, in combination with the finding that order effects were equally strong in both directions, suggests a rather simple explanation. In contrast to most other studies that investigate order effects, the two scenarios that we used in our experiment both contained the identical options of doing nothing and push (see above). Hence, participants who either chose doing nothing or push in the first scenario could also make the very same choice in the second scenario. A plausible hypothesis would thus be that participants' need for consistency in their judgments can account for the order effects we found. Since consistency is also an important philosophical value, this explanation might be especially fitting in case of our expert moral philosophers (see also Campbell \& Kumar, 2012)..$^{12}$

We will organize the remainder of our discussion around the two findings concerning expert participants that are most striking from the perspective of the expertise defense. First, expert participants were more likely to choose the push option when more options (6 versus 2) were available. Second, we also found order effects in expert ethicists in both

\footnotetext{
${ }^{12}$ See also footnote 9.
} 
directions, from Two Options to Six Options and vice versa, again concerning choices of the push option.

\subsection{Finding 1: The Influence of Irrelevant Options on Expert Judgments}

Concerning the expertise defense, the main significance of the effect of several options is that the additional intermediate options in Six Options arguably constitute an irrelevant factor in the experimental restrictionist sense, that is, a factor that is irrelevant to the proper moral evaluation of this case (in a sense to be further specified below). Nevertheless, our data provide suggestive evidence that the intuitive judgments of expert ethicists were influenced by the presence of additional intermediate options.

To be sure, the additional options are not per se irrelevant to the question which of the options in Six Options is morally optimal. Some moral philosophers might argue that one of the intermediate options is in fact morally better than either doing nothing or the push option (see, e.g., Kamm, 2007). A potential reason might be that Option 5, for example, still saves four lives in comparison to the nine people that would be killed in case of doing nothing, yet without using any of the victims as a means. Thus, there are prima facie morally relevant considerations that might justify preferring one of the intermediate options over both doing nothing and pushing. In that sense, the intermediate options are not an irrelevant factor concerning one's moral evaluation of the case. Without begging substantial philosophical questions, we therefore cannot say that the $22 \%$ of our expert participants who chose an intermediate option in Six Options were influenced by a morally irrelevant factor.

However, the significant increase in expert ratings for the push option in Six Options, when presented first, as compared to Two Options, when presented first, is the result of an irrelevant factor. For with respect to that difference, the presence of additional intermediate options is indeed morally irrelevant.

Why is the presence of additional intermediate options a morally irrelevant factor here? One's choice of either doing nothing or the push option in Two Options, if rational, is the result of a direct moral comparison of the two options in terms of the number of lives saved and their further morally relevant features, which are explicitly stipulated in the case description. Moreover, the option of doing nothing and the push option are exactly the same 
in Six Options, and thus both options are available for direct moral comparison in Six Options, just as they are in Two Options. The only morally relevant feature that actually speaks in favor of the push option is the number of lives saved (other morally relevant features rather tend to speak against the push option). In this respect, the push option clearly dominates all other options, because it would save the net sum of eight lives-more than any other option. Thus, the only defensible moral reason that would favor the push option over all other options - that it saves the most lives-applies to Six Options just as clearly and unambiguously as it applies to Two Options.

Therefore, if the choices of our expert participants were rational, and given the random assignment of expert participants to the two conditions, we would expect only two possible outcomes. First, we might get lower expert ratings for the push option in Six Options (when presented first) than in Two Options (when presented first) if some expert participants also attach moral weight to features other than the number of lives saved, for example, to the fact that someone is used as a mere means, and therefore choose an intermediate option over the push option in Six Options. Second, we might get basically the same expert ratings for the push option in Six Options (when presented first) as in Two Options (when presented first) if all expert participants who attach a decisive moral weight to the number of lives saved would do so in a purely consequentialist vein, that is, by ignoring all other features of the available options. What we would not expect, however, is a third kind of outcome, namely, that the expert ratings for the push option are significantly higher in Six Options (when presented first) than in Two Options (when presented first). For this would require that some expert participants who would not attach a decisive moral weight to the number of lives saved in Two Options, and thus opt for doing nothing in the two-option scenario, nevertheless do attach a decisive moral weight to the number of lives saved in Six Options, despite the fact that the option of doing nothing is equally available in the six-option scenario. Given that the push option dominates all other options in terms of the number of lives saved in both Two Options and Six Options, this would be an irrational outcome pattern in our group of expert participants. The fact that we nevertheless observed significantly higher expert ratings for the push option in Six Options (when presented first) than in Two Options (when presented first) thus indicates the workings of a morally irrelevant factor in our expert ethicists, namely, the presence of additional intermediate options, which is the only difference between the two cases. 
The rationale that underlies this argument is captured by the so-called regularity principle, which is a minimum condition in most existing models of rational choice (cf. Huber et al., 1982; Luce, 1959; Tversky, 1972). What the regularity principle says, in a nutshell, is that " $[. .$.$] the addition of an option to a choice set should never increase the probability of$ selecting an option from the original set" (Rieskamp, Busemeyer, \& Mellers, 2006, p. 644). Thus, adopting the regularity principle as a basic principle of rational choice generates an even stronger and more general reason why the intermediate options in Six Options work as an irrelevant factor in the experimental restrictionist sense: because any significant increase of the ratings for either pushing or doing nothing in Six Options (when presented first), in comparison to Two Options (when presented first), would thereby be rendered irrationalirrespective of one's particular first-order ethical views.

One possible objection might be that the additional intermediate options in Six Options make the morally relevant differences between the available options more salient (cf. Wiegmann \& Meyer, 2015), which may then justify a moral preference for the push option over doing nothing in Six Options. However, all information about the morally relevant features of doing nothing and the push option is equally present in both Two Options and Six Options, and so an increase of ratings for the push option in Six Options (when presented first) in comparison to Two Options (when presented first) would still constitute a rational shortcoming of some sort. Moreover, the morally relevant differences between doing nothing and the push option should already be maximally salient to our expert ethicists in particular, due to their many years of professional training in moral philosophy, where assessments of this kind are very much "routine business".

Another possible and even stronger objection might be that one can actually learn something new from the additional options in Six Options, and that this new information might justify a different moral assessment of the push option in direct comparison to doing nothing. ${ }^{13}$ But first, it is quite unclear which morally relevant fact about these two options one might learn from Six Options that one cannot also learn from Two Options. And second, it seems especially implausible that our expert ethicists would learn something new from Six Options that they did not already know before (see below for further discussion, where we call this the learning hypothesis).

\footnotetext{
${ }^{13}$ Thanks to Joshua Knobe for the objection.
} 


\subsection{Finding 2: Order Effects in Expert Judgments}

A second striking finding are the order effects in expert ethicists from Six Options to Two Options and vice versa with respect to the push option. As a reminder, when Two Options was presented first, a clear majority of expert participants judged that the agent should do nothing, whereas a narrow majority of expert participants preferred the push option when Two Options was presented after Six Options. When Six Options was presented first, the push option was preferred by half of all experts, whereas the majority preferred not to push when Six Options was presented after Two Options. This result is in line with the findings about order effects in expert ethicists by Schwitzgebel and Cushman $(2012,2015)$.

What are the implications of these order effects for the expertise defense against the experimental restrictionist challenge? The order in which Two Options and Six Options are presented seems irrelevant to their correct moral evaluation. Concerning our results, there is no good reason why one's verdict about Two Options should be different if one sees Six Options first or vice versa. Thus, our expert participants are susceptible to an irrelevant factor in the experimental restrictionist sense (see above), namely, order of presentation.

One might argue that the reversal of expert preferences in Two Options when presented first versus Two Options when presented after Six Options is not due to the influence of a morally irrelevant factor, but rather to the fact that the experts learned something new from Six Options, and then applied this new knowledge to the Two Options case (cf. Horne \& Livengood, 2015). Let us call this objection the learning hypothesis (cf. Unger, 1996; Weijers et al., ms).

Our first reply is that one would have to make a number of important specifications before the learning hypothesis can be properly assessed: it would have to be specified what exactly the experts might learn from their exposure to Six Options, and why this newly acquired knowledge might justify higher expert ratings for the push option in Two Options.

Apart from that, the fact that the order effect was not stronger in lay people than in expert ethicists speaks against the learning hypothesis. For if there really were something to be learned from Six Options, one would expect that our lay participants-who had no prior experience with trolley-style cases, and presumably much less experience with moral 
problem cases in general-would learn something new, rather than our expert participants, who should already be highly familiar with the relevant moral considerations and scenarios.

However, the strongest objection to the learning hypothesis is that we did not only find an order effect in the direction from Six Options to Two Options, but an equally strong effect in the reverse direction from Two Options to Six Options. Since Two Options contains much less information or potential knowledge than Six Options, it seems quite implausible that one can learn something new by first considering Two Options that would justify the subsequent pattern of expert ratings in Six Options.

\section{Conclusion}

In the experiment reported in this paper, we investigated the moral intuitions of expert participants and lay participants concerning two questions.

First, we tested whether adding intermediate options to the push dilemma would increase the proportion of participants who prefer the push option. While there is suggestive evidence for the effect in general (all participants taken together) and for expert participants in particular, there was only a non-significant trend for lay participants.

Second, we tested whether presenting the classic two-option version of the push dilemma and the six-option version in varying order has an effect on participants' intuitive judgments. When the two-option case was presented after the six-option case, the number of participants who chose the push option increased significantly, as compared to the condition in which the two-option case was presented first. And when the six-option case was presented after the two-option case, the number of participants who preferred the push option decreased significantly, as compared to the condition in which the six-option case was presented first. Again, these results hold for all participants taken together and also for expert participants, while there was only a non-significant trend for lay participants.

In descriptive terms, both kinds of effects-the effect of additional intermediate options and the effect of order of presentation-were stronger in expert participants than in lay participants, but this expert-lay difference itself was not statistically significant. For this reason, we only made the conservative assumption that both effects were not weaker in experts than in lay people. We then argued that both effects are due to the influence of a 
morally irrelevant factor-irrelevant options and order of presentation-which has

unfavorable implications for the expertise defense against the experimental restrictionist

challenge. In line with previous research, we found no evidence that the intuitive judgments of philosophical experts are more resistant to the influence of irrelevant factors than those of lay people. ${ }^{14}$

\section{References:}

Alexander, J. (2012). Experimental Philosophy: An Introduction. Cambridge: Polity Press.

Alexander, J., Mallon, R., \& Weinberg, J. (2009). Accentuate the Negative. Review of Philosophy and Psychology, 1(2), 297-314. https://doi.org/10.1007/s13164-009-0015-2

Alexander, J., \& Weinberg, J. (2007). Analytic Epistemology and Experimental Philosophy. Philosophy Compass, 2, 56-80.

Beebe, J. R., \& Monaghan, J. (2018). Epistemic Closure in Folk Epistemology. In T. Lombrozo, J. Knobe, \& S. Nichols (Eds.), Oxford Studies in Experimental Philosophy, Volume Two (pp. 38-70). New York: Oxford University Press.

Bengson, J. (2015). The Intellectual Given. Mind, 124(495), 707-760.

https://doi.org/10.1093/mind/fzv029

Buckwalter, W. (2016). Intuition Fail: Philosophical Activity and the Limits of Expertise. Philosophy and Phenomenological Research, 92(2), 378-410. https://doi.org/10.1111/phpr.12147

\footnotetext{
${ }^{14}$ We would like to thank James Beebe, Eugen Fischer, Bryan Frances, York Hagmayer, Hanjo Hamann, Matthias Katzer, Antti Kauppinen, Markus Kneer, Joshua Knobe, Victor Kumar, Clayton Littlejohn, Dustin Locke, Edouard Machery, Angra Mainyu, Jonas Nagel, Shaun Nichols, Philip Nickel, Sven Nyholm, Jana Samland, Hanno Sauer, Andreas Spahn, Pascale Willemsen, Michael Waldmann, and several anonymous reviewers for very helpful comments and discussions. Many thanks also to our audiences at the Philosophy \& Ethics Departmental Seminar at Eindhoven University of Technology in June 2016, at the Bonn/Cologne Research Colloquium on Epistemology at University of Cologne in June 2016, at the Legal Studies \& Ethics Department of the Frankfurt School of Finance \& Management in October 2016, at the ZiF Workshop "Is and ought: The Ethical and Legal Relevance of Moral Psychology "at the Zentrum für interdisziplinäre Forschung (ZiF) of University of Bielefeld in May 2017, at the workshop The Experimental Philosophy of Morality and Causation - Perspectives from Philosophy, Psychology, and Law of the Experimental Philosophy Group Germany at Ruhr-University Bochum in June 2017, at The Eighth (2017) Workshop of the Experimental Philosophy Group (UK) at University of East Anglia in July 2017. Alex Wiegmann's work on this paper was supported by a grant of Deutsche Forschungsgemeinschaft (The Psychology of Moral Dilemmas, project number 167159114). Joachim Horvath's work on this paper was supported by a grant of Deutsche Forschungsgemeinschaft for the Emmy Noether Independent Junior Research Group Experimental Philosophy and the Method of Cases: Theoretical Foundations, Responses, and Alternatives (EXTRA), project number 391304769.
} 
Campbell, R., \& Kumar, V. (2012). Moral Reasoning on the Ground. Ethics, 122(2), 273-312.

https://doi.org/10.1086/663980

Cao, F., Zhang, J., Song, L., Wang, S., Miao, D., \& Peng, J. (2017). Framing Effect in the Trolley Problem and Footbridge Dilemma: Number of Saved Lives Matters. Psychological Reports, 120(1), 88-101. https://doi.org/10.1177/0033294116685866

Cappelen, H. (2012). Philosophy Without Intuitions. Oxford: Oxford University Press.

Capraro, V., \& Sippel, J. (2017). Gender Differences in Moral Judgment and the Evaluation of GenderSpecified Moral Agents. Cognitive Processing, 18(4), 399-405. https://doi.org/10.1007/s10339-0170822-9

Carter, J. A., Peterson, M., \& Bezooijen, B. van. (2015). Not Knowing a Cat is a Cat: Analyticity and Knowledge Ascriptions. Review of Philosophy and Psychology, 1-18. https://doi.org/10.1007/s13164015-0279-7

Carter, J. A., Pritchard, D., \& Sheperd, J. (ms). Knowledge-How, Understanding-Why and Epistemic Luck: An Experimental Study.

Chudnoff, E. (2013). Intuition. Oxford: Oxford University Press.

Crandall, B., \& Getchell-Reiter, K. (1993). Critical Decision Method: A Technique for Eliciting Concrete Assessment Indicators from the Intuition of NICU Nurses. Advances in Nursing Science, 16(1), 42-51. https://doi.org/10.1097/00012272-199309000-00006

De Cruz, H. (2015). Where Philosophical Intuitions Come From. Australasian Journal of Philosophy, 93(2), 233-249. https://doi.org/10.1080/00048402.2014.967792

Deutsch, M. (2015). The Myth of the Intuitive: Experimental Philosophy and Philosophical Method. Cambridge, Mass.: MIT Press.

Devitt, M. (2011). Experimental Semantics. Philosophy and Phenomenological Research, 82(2), 418435. https://doi.org/10.1111/j.1933-1592.2010.00413.x

Ericsson, K. A., Charness, N., Feltovich, P., \& Hoffman, R. (Eds.). (2006). The Cambridge Handbook of Expertise and Expert Performance. New York: Cambridge University Press.

Feltz, A., \& Cokely, E. (2009). Do Judgments About Freedom and Responsibility Depend on Who You Are? Personality Differences in Intuitions About Compatibilism and Incompatibilism. Consciousness and Cognition, 18(1), 342-350. https://doi.org/10.1016/j.concog.2008.08.001

Feltz, A., \& Cokely, E. (2012). The Philosophical Personality Argument. Philosophical Studies, 161(2), 227-246. https://doi.org/10.1007/s11098-011-9731-4

Hales, S. (2006). Relativism and the Foundations of Philosophy. Cambridge, Mass.: MIT Press.

Hitchcock, C. (2012). Thought Experiments, Real Experiments, and the Expertise Objection. European Journal for Philosophy of Science, 2(2), 205-218. https://doi.org/10.1007/s13194-012-0051-0

Hitchcock, C., \& Knobe, J. (2009). Cause and Norm. Journal of Philosophy, 106(11), 587-612. 
Hofmann, F. (2010). Intuitions, Concepts, and Imagination. Philosophical Psychology, 23(4), 529-546. https://doi.org/10.1080/09515089.2010.505980

Hooker, B. (2000). Ideal Code, Real World: A Rule-Consequentialist Theory of Morality. Oxford: Oxford University Press.

Horne, Z., \& Livengood, J. (2015). Ordering Effects, Updating Effects, and the Specter of Global Skepticism. Synthese, 1-30. https://doi.org/10.1007/s11229-015-0985-9

Horne, Z., Powell, D., \& Spino, J. (2013). Belief Updating in Moral Dilemmas. Review of Philosophy and Psychology, 4(4), 705-714. https://doi.org/10.1007/s13164-013-0159-y

Horvath, J. (2010). How (Not) to React to Experimental Philosophy. Philosophical Psychology, 23(4), $447-480$.

Horvath, J., \& Wiegmann, A. (2016). Intuitive Expertise and Intuitions about Knowledge. Philosophical Studies, 173(10), 2701-2726. https://doi.org/10.1007/s11098-016-0627-1

Huber, J., Payne, J. W., \& Puto, C. (1982). Adding Asymmetrically Dominated Alternatives: Violations of Regularity and the Similarity Hypothesis. Journal of Consumer Research, 9(1), 90-98.

https://doi.org/10.1086/208899

Kahneman, D., \& Klein, G. (2009). Conditions for Intuitive Expertise: A Failure to Disagree. American Psychologist, 64(6), 515-526. https://doi.org/10.1037/a0016755

Kamm, F. M. (1998). Grouping and the Imposition of Loss. Utilitas, 10(3), 292-.

Kamm, F. M. (2007). Intricate Ethics: Rights, Responsibilities, and Permissible Harm. New York: Oxford University Press.

Kauppinen, A. (2007). The Rise and Fall of Experimental Philosophy. Philosophical Explorations, 10(2), 95-118.

Klein, G., Calderwood, R., \& Clinton-Cirocco, A. (1986). Rapid Decision Making on the Fire Ground. Proceedings of the Human Factors and Ergonomics Society Annual Meeting, 30(6), 576-580. https://doi.org/10.1177/154193128603000616

Liao, S. M., Wiegmann, A., Alexander, J., \& Vong, G. (2012). Putting the Trolley in Order: Experimental Philosophy and the Loop Case. Philosophical Psychology, 25(5), 661-671.

https://doi.org/10.1080/09515089.2011.627536

Lombrozo, T. (2009). The Role of Moral Commitments in Moral Judgment. Cognitive Science, 33(2), 273-286. https://doi.org/10.1111/j.1551-6709.2009.01013.x

Luce, R. D. (1959). Individual Choice Behavior: A Theoretical Analysis. New York: Wiley.

Ludwig, K. (2007). The Epistemology of Thought Experiments: First Person versus Third Person Approaches. Midwest Studies In Philosophy, 31(1), 128-159.

Machery, E. (2012). Expertise and Intuitions About Reference. Theoria, 27(1), 37-54.

Machery, E. (2017). Philosophy Within Its Proper Bounds. Oxford: Oxford University Press. 
Machery, E., Mallon, R., Nichols, S., \& Stich, S. (2004). Semantics, Cross-Cultural Style. Cognition, 92, $1-12$.

Machery, E., \& O'Neill, E. (Eds.). (2014). Current Controversies in Experimental Philosophy. London: Routledge.

Mikhail, J. (2007). Universal Moral Grammar: Theory, Evidence and the Future. Trends in Cognitive Sciences, 11(4), 143-152. https://doi.org/10.1016/j.tics.2006.12.007

Nado, J. (2014). Philosophical Expertise. Philosophy Compass, 9(9), 631-641.

https://doi.org/10.1111/phc3.12154

Nado, J. (2015). Philosophical Expertise and Scientific Expertise. Philosophical Psychology, 28(7), 1026-1044. https://doi.org/10.1080/09515089.2014.961186

Nado, J. (2016). The Intuition Deniers. Philosophical Studies, 173(3), 781-800.

https://doi.org/10.1007/s11098-015-0519-9

Nagel, J. (2012). Intuitions and Experiments: A Defense of the Case Method in Epistemology. Philosophy and Phenomenological Research, 85(3), 495-527. https://doi.org/10.1111/j.19331592.2012.00634.x

Nichols, S., \& Knobe, J. (2007). Moral Responsibility and Determinism: The Cognitive Science of Folk Intuitions. Noûs, 41(4), 663-685.

Palan, S., \& Schitter, C. (2017). Prolific.ac-A Subject Pool for Online Experiments. Journal of Behavioral and Experimental Finance. https://doi.org/10.1016/j.jbef.2017.12.004

Rieskamp, J., Busemeyer, J. R., \& Mellers, B. A. (2006). Extending the Bounds of Rationality: Evidence and Theories of Preferential Choice. Journal of Economic Literature, 44(3), 631-661.

https://doi.org/10.1257/jel.44.3.631

Rini, R. A. (2014). Analogies, Moral Intuitions, and the Expertise Defence. Review of Philosophy and Psychology, 5(2), 169-181. https://doi.org/10.1007/s13164-013-0163-2

Rini, R. A. (2015). How Not to Test for Philosophical Expertise. Synthese, 192(2), 431-452.

https://doi.org/10.1007/s11229-014-0579-y

Schulz, E., Cokely, E., \& Feltz, A. (2011). Persistent Bias in Expert Judgments About Free Will and Moral Responsibility: A Test of the Expertise Defense. Consciousness and Cognition, 20(4), 17221731. https://doi.org/10.1016/j.concog.2011.04.007

Schwitzgebel, E., \& Cushman, F. (2012). Expertise in Moral Reasoning? Order Effects on Moral Judgment in Professional Philosophers and Non-Philosophers. Mind \& Language, 27(2), 135-153. https://doi.org/10.1111/j.1468-0017.2012.01438.x

Schwitzgebel, E., \& Cushman, F. (2015). Philosophers' Biased Judgments Persist Despite Training, Expertise and Reflection. Cognition, 141, 127-137. https://doi.org/10.1016/j.cognition.2015.04.015

Sorensen, R. (2014). Novice Thought Experiments. In A. R. Booth \& D. P. Rowbottom (Eds.), Intuitions (pp. 135-147). Oxford: Oxford University Press. 
Sosa, E. (2007). Experimental Philosophy and Philosophical Intuition. Philosophical Studies, 132(1), 99-107.

Sosa, E. (2009). A Defense of the Use of Intuitions in Philosophy. In M. Bishop \& D. Murphy (Eds.), Stich and his Critics (pp. 101-112). Oxford: Blackwell.

Swain, S., Alexander, J., \& Weinberg, J. (2008). The Instability of Philosophical Intuitions: Running Hot and Cold on Truetemp. Philosophy and Phenomenological Research, 76(1), 138-155.

Sytsma, J., \& Livengood, J. (2016). The Theory and Practice of Experimental Philosophy. Peterborough, Ont.: Broadview Press.

Sytsma, J., \& Machery, E. (2010). Two Conceptions of Subjective Experience. Philosophical Studies, 151(2), 299-327. https://doi.org/10.1007/s11098-009-9439-x

Thomson, J. J. (1985). The Trolley Problem. The Yale Law Journal, 94(6), 1395-1415.

https://doi.org/10.2307/796133

Tobia, K., Buckwalter, W., \& Stich, S. (2013). Moral Intuitions: Are Philosophers Experts? Philosophical Psychology, 26(5), 629-638. https://doi.org/10.1080/09515089.2012.696327

Tobia, K., Chapman, G., \& Stich, S. (2013). Cleanliness is Next to Morality, Even for Philosophers. Journal of Consciousness Studies, 20(11-12), 195-204.

Tversky, A. (1972). Elimination by Aspects: A Theory of Choice. Psychological Review, 79(4), 281-299.

Unger, P. (1992). Causing and Preventing Serious Harm. Philosophical Studies, 65(3), 227-255. https://doi.org/10.1007/BF00354612

Unger, P. (1996). Living High and Letting Die: Our Illusion of Innocence. New York: Oxford University Press.

Waldmann, M. R., Nagel, J., \& Wiegmann, A. (2012). Moral Judgment. In K. J. Holyoak \& R. G. Morrison (Eds.), The Oxford Handbook of Thinking and Reasoning (pp. 364-386). Oxford: Oxford University Press.

Weijers, D. (2015). To Push or Not to Push? Should That be the Question? Retrieved February 28, 2018, from http://www.danceofreason.com/2015/02/to-push-or-not-to-push-should-that-be.html

Weijers, D., Unger, P., \& Sytsma, J. (ms). Trolley Problems Reconsidered: Testing the Method of Several Options.

Weinberg, J., Gonnerman, C., Buckner, C., \& Alexander, J. (2010). Are Philosophers Expert Intuiters? Philosophical Psychology, 23(3), 331-355.

Wiegmann, A., \& Meyer, K. (2015). When Killing the Heavy Man Seems Right. Making People Utilitarian by Simply Adding Options to Moral Dilemmas. In D. C. Noelle, R. Dale, A. S. Warlaumont, J. Yoshimi, T. Matlock, C. D. Jennings, \& P. P. Maglio (Eds.), Proceedings of the 37th Annual Meeting of the Cognitive Science Society. Austin, TX: Cognitive Science Society.

Wiegmann, A., \& Waldmann, M. R. (2014). Transfer Effects Between Moral Dilemmas: A Causal Model Theory. Cognition, 131(1), 28-43. https://doi.org/10.1016/j.cognition.2013.12.004 
Williamson, T. (2005). Armchair Philosophy, Metaphysical Modality and Counterfactual Thinking. Proceedings of the Aristotelian Society, 105(1), 1-23.

Williamson, T. (2007). The Philosophy of Philosophy. Malden, Mass.: Blackwell.

Williamson, T. (2011). Philosophical Expertise and the Burden of Proof. Metaphilosophy, 42(3), 215229. https://doi.org/10.1111/j.1467-9973.2011.01685.x 